\title{
Distinguishing Isotopes in the Electron Microscope: In-situ TEM-SIMS Correlative Analysis
}

\author{
Lluís Yedra ${ }^{1}$, Santhana Eswara ${ }^{1}$, David Dowsett ${ }^{1}$ and Tom Wirtz ${ }^{1}$
}

1. Advanced Instrumentation for Ion Nano-Analytics (AINA), MRT Department, Luxembourg Institute of Science and Technology (LIST), 41 rue du Brill, Belvaux, Luxembourg

While Transmission Electron Microscopy (TEM) offers superior resolution and a variety of analytical capabilities, isotopic or light element analyses remain out of reach for Energy Dispersive Spectroscopy (EDS) or Electron Energy-Loss Spectroscopy (EELS) [1]. However, these analyses count among the advantages of secondary Ion Mass Spectrometry (SIMS), which also provides high chemical sensitivity and high dynamical range, but offers poor lateral resolution [2]. To advance the frontiers of science, it is thus necessary to complement the strengths of SIMS with a high-resolution imaging technique such as TEM. As ex-situ approaches are prone to sample modification artifacts, we have developed an in-situ combination of TEM and SIMS called the Parallel Ion-Electron Spectrometry (PIES).

The prototype instrument [3] shown in Fig. 1, consists of a Tecnai F20 with modified octagon and pole pieces to accommodate a FEI Magnum $\mathrm{Ga}^{+}$Focused Ion Beam (FIB) primary column and the secondary ion extraction optics. The extraction optics (extraction efficiency up to 90\%), are coupled with a compact double focusing magnetic-sector mass spectrometer developed in house. A special sample holder, which can be biased to high voltages $( \pm 4.5 \mathrm{kV})$ is used as part of the ion extraction system.

To demonstrate the potential of the analytical capabilities of the PIES methodology, lithium carbonate $\left(\mathrm{Li}_{2} \mathrm{CO}_{3}\right)$ was chosen. .Lithium exists in two stable isotopes in nature $\left({ }^{6} \mathrm{Li}\right.$ and $\left.{ }^{7} \mathrm{Li}\right)$ with very different abundances, $7.5 \%$ and $92.5 \%$, respectively. A powder sample of $\mathrm{Li}_{2} \mathrm{CO}_{3}$ enriched in ${ }^{6} \mathrm{Li}$ up to $95 \%$ was mixed with a natural-abundance sample. The goal of the investigation has been the distinction of particles according to their isotopic abundance.

Two different approaches arise from the TEM-SIMS correlation. On the one hand, for samples in which the areas of interest depend on the structure, TEM can be used for identification of those areas and SIMS will provide the analysis afterwards. On the other hand, for samples marked with isotopic (or elemental) labels, SIMS reveals the area of interest to be structurally analyzed by TEM. After acquiring mass spectra from each of the individual materials before mixing, mass filtered images of the mixed sample were obtained. Subsequent TEM allows for high resolution imaging of the same area, so that the particles can be identified as belonging to one of the two original materials according to the isotopic label as shown in Fig. 2. The strategies to correct imaging artifacts such as elongation, rotation and shear will be discussed and demonstrated with TEM-SIMS datasets.

The correlative approach leads to multimodal imaging of the same Regions-of-Interest (RoI). To fully exploit these results, image-fusion methods are employed to combine different channels of information. The fused PIES image then contains high spatial resolution and isotopic information..

In this presentation, we will introduce, discuss and demonstrate the technique and the correlative strategies for applications in the fields of materials and life sciences. 


\section{References:}

[1] R. F. Egerton, "Electron Energy-Loss Spectroscopy in the Electron Microscope” (3rd edition), (Springer, New York, 2011)

[2] A. Benninghoven, H. W. Werner, F. G. Rüdenauer, "Secondary ion mass spectrometry: Basic concepts, instrumental aspects, applications and trends" (Wiley-Interscience, New York, Chichester, Brisbane, Toronto, Singapore, 1987)

[3] T. Wirtz, et al, Nanotechnology 26, 434001 (2015)

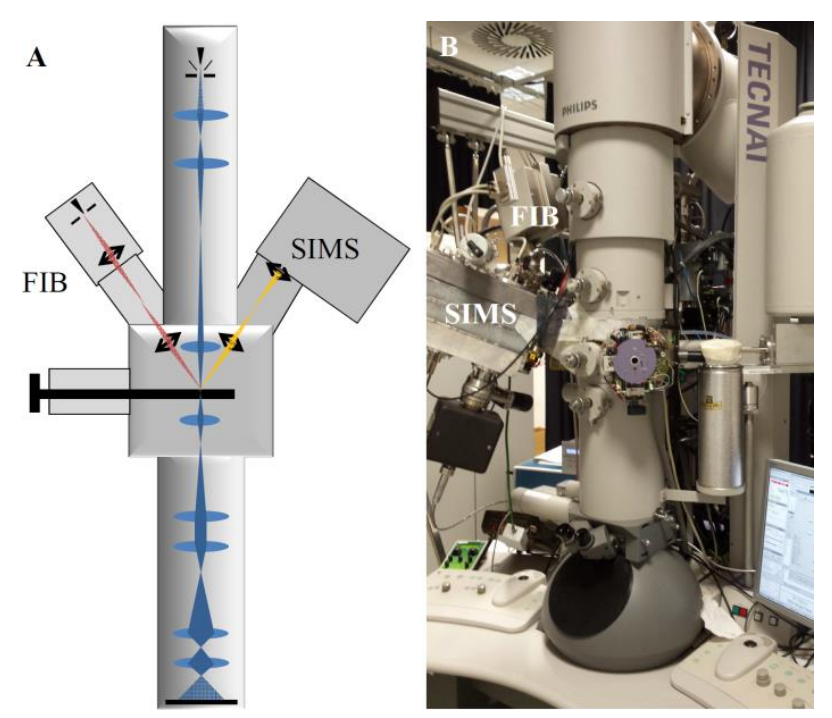

Figure 1: (A) Schematic and (B) Photo of the PIES instrument

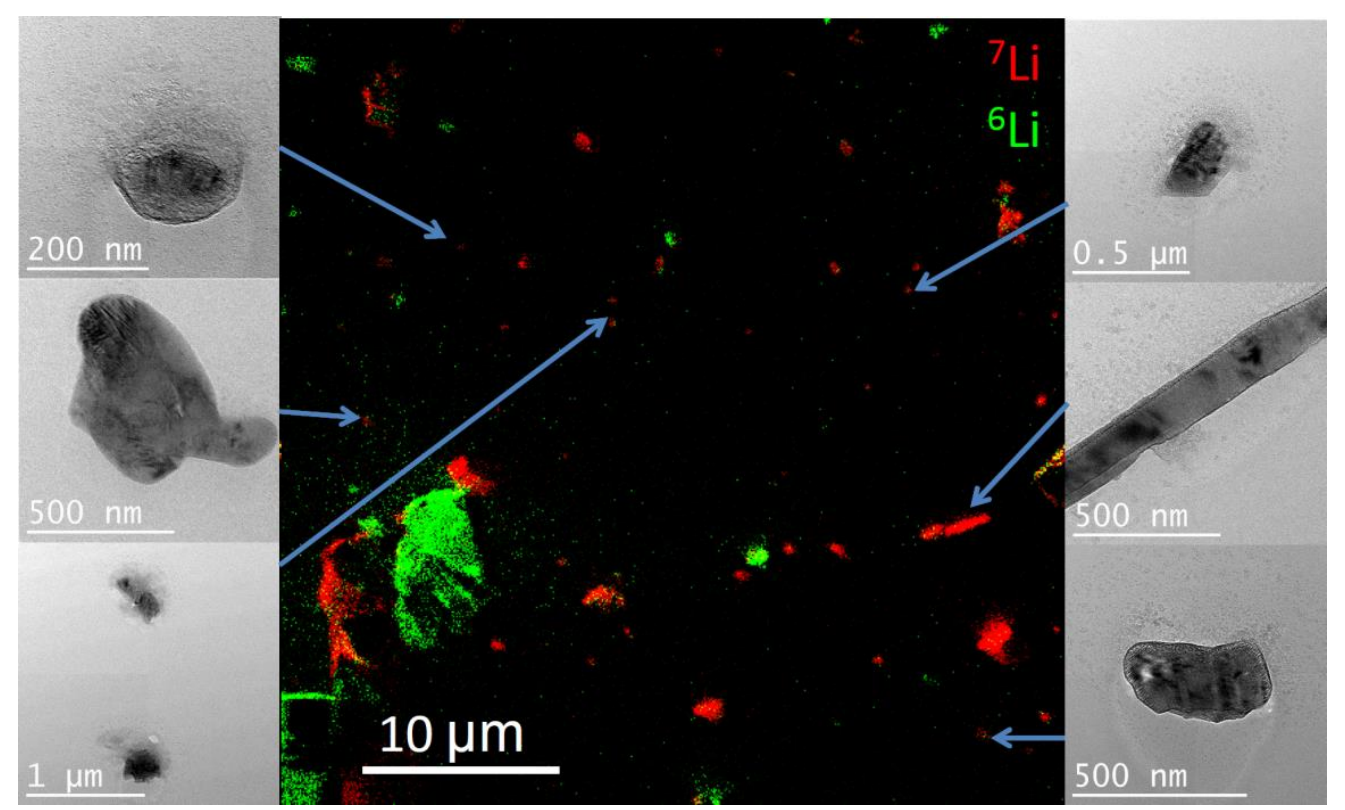

Figure 2: (Center) SIMS image depicting the ${ }^{6} \mathrm{Li}^{+}$and ${ }^{7} \mathrm{Li}^{+}$content of the mixed powder sample. The presence of two samples with different isotope abundances can be observed. (Left and Right) Bright Field TEM images of some of the particles, indicated by the blue arrows. 